\title{
Sensitivity of Dual Waveguide Probe Complex Permittivity and Permeability Measurement to Probe Lift-Off Error
}

\author{
M. J. Havrilla and M. W. Hyde IV ${ }^{* \dagger}$
}

\begin{abstract}
The effect of lift-off (i.e., air-gap) error in dual waveguide probe material characterization measurements is presented here. The mitigation of this error using a two-layer dyadic Green's function analysis is also discussed. Experimental results for gap scenarios of an electromagnetic shielding material are presented for validation purposes.
\end{abstract}

\section{INTRODUCTION}

Coaxial and waveguide probes have been studied for the past 30 years. Their uses include nondestructive material evaluation, subsurface crack detection, and even hyperthermia treatment [1]-[4]. Existing research has focused on obtaining the reflection coefficient, using a single probe, of an unknown material backed by a dielectric half-space or a perfect electric conductor (PEC). While this scenario is ideal for determining the dielectric constant of an unknown material, it suffers when one wants to also determine permeability because it lacks two (or more) independent measurements.

Several authors have developed techniques - most notably, two-thickness method, sample added method, and frequency varying method-to fill this single waveguide probe shortfall. These techniques, however, are not always applicable. A more suitable alternative has been proposed by Stewart [5]. The sensor, called a dual waveguide probe (Fig. 1, with lift-off gap), uses two waveguides to simultaneously measure the reflection and transmission coefficients thereby ensuring two independent measurements of the material under test (MUT).

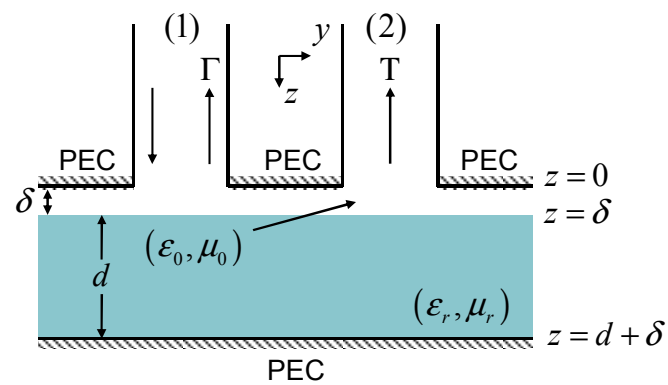

Figure 1: Dual probe geometry with lift-off $\delta$.
The sensitivity of the apparatus to waveguide alignment, MUT thickness, and flange thickness has been investigated [6]. One aspect of the dual waveguide probe that has yet to be investigated is its sensitivity to an air gap between the flange and the MUT (also known as probe lift-off error). The research presented in this paper investigates the dual waveguide probe's sensitivity to such a measurement error. The paper will include theoretical development of the method, mainly application of Love's Equivalence Principle and the Method of Moments (MoM). It will also include a brief derivation of the two-layer, parallel-plate dyadic Green's function (magnetic current excitation) for gap-error mitigation, which to the authors' knowledge does not appear in the literature. Lastly, measurement results will be shown demonstrating the sensitivity of permittivity and permeability measurements (obtained using the dual waveguide probe) to air gaps.

\section{MFIE FORMULATION AND SOLUTION}

Computation of the relative permittivity $\varepsilon_{r}$ and relative permeability $\mu_{r}$ of a PEC-backed shielding material (see Fig. 1) at angular frequency $\omega$ can be achieved by solving the following coupled equations

$$
\begin{aligned}
& \left|S_{11}^{\text {thy }}\left(\omega, \varepsilon_{r}, \mu_{r}\right)-S_{11}^{\exp }(\omega)\right|<t o l \\
& \left|S_{21}^{\text {thy }}\left(\omega, \varepsilon_{r}, \mu_{r}\right)-S_{21}^{\exp }(\omega)\right|<t o l
\end{aligned}
$$

The permittivity and permeability in (1) are iterated using a root-search algorithm (e.g., Newton's method) until the theoretical scattering parameters $S_{11}^{\text {thy }}=\Gamma, S_{21}^{\text {thy }}=\mathrm{T}$ match the experimental $/$ measured values $S_{11}^{\exp }, S_{21}^{\exp }$ to within specified tolerance tol (typically $1 \times 10^{-7}$ ). The experimental scattering parameters are easily obtained via network analyzer measurement and the corresponding theoretical values can be found using a magnetic field integral equation (MFIE) formulation, as discussed below.

\footnotetext{
* Department of Electrical and Computer Engineering, Air Force Institute of Technology, 2950 Hobson Way, WPAFB, OH 45433, USA, e-mail: milo.hyde@afit.edu and michael.havrilla@afit.edu, tel.: 937-255-3636, fax: 937-656-7061.

${ }^{\dagger}$ The views expressed in this article are those of the authors and do not reflect the official policy or position of the United States Air Force, Department of Defense, or the U.S. Government.
} 
The apertures at $z=0$ in guide 1 and 2 of Figure 1 can be replaced by equivalent magnetic surface currents $\vec{M}_{1}$ and $\vec{M}_{2}$, respectively, using Love's equivalence principle [7]. These equivalent currents emanate into the waveguide and parallel-plate regions with transverse magnetic fields given by [6]

$$
\begin{gathered}
\vec{H}_{t, w g}^{(1)}=\vec{h}_{1}^{(1)} e^{-\gamma_{1} z}-\sum_{n=1}^{N} \Gamma_{n} \vec{h}_{n}^{(1)} e^{\gamma_{n} z} \\
\vec{H}_{t, w g}^{(2)}=-\sum_{n=1}^{N} \mathrm{~T}_{n} \vec{h}_{n}^{(2)} e^{\gamma_{n} z} \\
\vec{H}_{t}^{p p}=\frac{\left(k^{2}+\nabla_{t} \nabla \cdot\right)}{j \omega \mu} \sum_{q=1}^{2} \int_{S_{q}} \vec{G}\left(\vec{r} \mid \vec{r}^{\prime}\right) \cdot \vec{M}_{q}\left(\vec{r}^{\prime}\right) d S^{\prime} .
\end{gathered}
$$

In (2)-(4), $\gamma_{n}$ is the $n^{\text {th }}$-mode propagation constant, $\vec{h}_{n}^{(1),(2)}$ are the normal modes in guide 1 and 2 (with $\vec{h}_{1}$ representing the dominant $T E_{10}$ mode), $\vec{G}$ is the two-layer parallel-plate dyadic Green's function (developed in the next section) and $\Gamma_{n}, \mathrm{~T}_{n}$ are the $n^{\text {th }}$-mode reflection and transmission coefficients, respectively. Note, using orthogonality of the normal modes, the reflection and transmission coefficients are related to $\vec{M}_{1}, \vec{M}_{2}$ via the relations

$$
\begin{gathered}
\Gamma_{n}=\int_{S_{1}} \vec{e}_{n}^{(1)} \cdot \hat{n} \times \vec{M}_{1} d S-\delta_{n 1}, \delta_{n 1}=\left\{\begin{array}{l}
1 \\
\ldots . n=1 \\
0 \ldots n \neq 1
\end{array}\right. \\
\mathrm{T}_{n}=\int_{S_{2}} \vec{e}_{n}^{(2)} \cdot \hat{n} \times \vec{M}_{2} d S, \\
\vec{e}_{n}^{(1),(2)}=-Z_{n} \hat{n} \times \vec{h}_{n}^{(1),(2)}
\end{gathered}
$$

where $Z_{n}$ is the $n^{\text {th }}$-mode wave impedance given in [6]-[7] and $\hat{n}=\hat{z}$. It is important to recognize that the same equivalent currents were used in the waveguide and parallel-plate regions since the tangential electric fields at the apertures are continuous in the original geometry of Figure 1.

The coupled MFIEs are formulated by equating the tangential magnetic fields at aperture 1 and 2, namely

$$
\begin{aligned}
& \vec{h}_{1}^{(1)}(\vec{r})-\sum_{n=1}^{N} \Gamma_{n} \vec{h}_{n}^{(1)}(\vec{r})= \\
& \quad \frac{\left(k^{2}+\nabla_{t} \nabla \cdot\right)}{j \omega \mu} \sum_{q=1}^{2} \int_{S_{q}} \vec{G}\left(\vec{r} \mid \vec{r}^{\prime}\right) \cdot \vec{M}_{q}\left(\vec{r}^{\prime}\right) d S^{\prime} \ldots \vec{r} \in S_{1} \\
& -\sum_{n=1}^{N} \mathrm{~T}_{n} \vec{h}_{n}^{(2)}(\vec{r})= \\
& \frac{\left(k^{2}+\nabla_{t} \nabla \cdot\right)}{j \omega \mu} \sum_{q=1}^{2} \int_{S_{q}} \vec{G}\left(\vec{r} \mid \vec{r}^{\prime}\right) \cdot \vec{M}_{q}\left(\vec{r}^{\prime}\right) d S^{\prime} \ldots \vec{r} \in S_{2} .
\end{aligned}
$$

These coupled MFIEs can be solved using the MoM by first expanding the unknown currents in terms of the waveguide electric field normal modes and testing with the magnetic field normal modes

$$
\begin{gathered}
\vec{M}_{1}=\sum_{n=1}^{N} c_{n}^{(1)} \vec{e}_{n}^{(1)}, \vec{M}_{2}=\sum_{n=1}^{N} c_{n}^{(2)} \vec{e}_{n}^{(2)} \\
\int_{S_{1}} \vec{h}_{n}^{(1)} \cdot\{(8)\} d S, \int_{S_{2}} \vec{h}_{n}^{(2)} \cdot\{(9)\} d S \ldots m=1, \ldots, N,
\end{gathered}
$$

leading to the $2 N \times 2 N$ partitioned matrix equation given in [6] by

$$
\left[\begin{array}{ll}
A^{(1)(1)} & A^{(1)(2)} \\
A^{(2)(1)} & A^{(2)(2)}
\end{array}\right]\left[\begin{array}{l}
c^{(1)} \\
c^{(2)}
\end{array}\right]=\left[\begin{array}{l}
b^{(1)} \\
b^{(2)}
\end{array}\right]
$$

Note, solution of this matrix equation leads to the final desired result

$$
S_{11}^{\text {thy }}=\Gamma=\Gamma_{1}=c_{1}^{(1)}-1, S_{21}^{\text {thy }}=\mathrm{T}=\mathrm{T}_{1}=c_{1}^{(2)} .
$$

The derivation of the two-layer parallel-plate dyadic Green's function for air-gap mitigation is developed next to complete the theoretical analysis.

\section{GREEN'S FUNCTION DEVELOPMENT}

The two-layer dyadic Green's function for a magnetic current immersed in the air gap of the parallel-plate region shown in Figure 1 can be found by solving the vector potential wave equations (for $\alpha=x, y, z$ )

$$
\begin{gathered}
\nabla^{2} F_{a \alpha}+k_{0}^{2} F_{a \alpha}=-\varepsilon_{0} M_{\alpha} \quad \ldots \vec{r} \in(0<z<\delta) \\
\nabla^{2} F_{m \alpha}+k^{2} F_{m \alpha}=0 \quad \ldots \vec{r} \in(\delta<z<d+\delta)
\end{gathered}
$$

and enforcing appropriate boundary conditions at the $\operatorname{PEC}(z=0, d+\delta)$ and material $(z=\delta)$ interfaces.

Since the parallel-plate structure is infinite in extent and invariant along the $x, y$ directions, the mathematical analysis can be simplified by utilizing the generic Fourier transform pair

$$
\begin{gathered}
\tilde{\Psi}(\xi, \eta)=\int_{-\infty}^{\infty} \int_{-\infty}^{\infty} \Psi(x, y) e^{-j \xi x} e^{-j \eta y} d x d y \\
\Psi(x, y)=\frac{1}{(2 \pi)^{2}} \int_{-\infty}^{\infty} \int_{-\infty}^{\infty} \tilde{\Psi}(\xi, \eta) e^{j \xi x} e^{j \eta y} d \eta d \xi
\end{gathered}
$$

Invoking (15), the general solution to (14) is [6]

$$
\begin{gathered}
\tilde{F}_{a \alpha}=\int_{z^{\prime}} \frac{e^{-p_{a}\left|z-z^{\prime}\right|} \varepsilon_{0} \tilde{M}_{\alpha}}{2 p_{a}} d z^{\prime}+W_{a \alpha}^{+} e^{-p_{a} z}+W_{a \alpha}^{-} e^{p_{a} z} \\
\tilde{F}_{m \alpha}=W_{m \alpha}^{+} e^{-p_{m} z}+W_{m \alpha}^{-} e^{p_{m} z}
\end{gathered}
$$


where $p_{a}=\sqrt{\xi^{2}+\eta^{2}-k_{0}^{2}}$ and $p_{m}=\sqrt{\xi^{2}+\eta^{2}-k^{2}}$ Computing the electric and magnetic fields in terms of the vector potential [7] relations in (16) and enforcing the boundary conditions

$$
\begin{aligned}
& \hat{n} \times \vec{E}_{a}=\hat{n} \times \vec{E}_{m}, \hat{n} \times \vec{H}_{a}=\hat{n} \times \vec{H}_{m} \ldots z=\delta \\
& \hat{n} \times \vec{E}_{a}=0 \quad \ldots z=0, \hat{n} \times \vec{E}_{m}=0 \quad \ldots z=d+\delta
\end{aligned}
$$

produces the desired result

$$
\begin{aligned}
& \vec{G}=\hat{x} G_{x x} \hat{x}+\hat{y} G_{y y} \hat{y}+\hat{z} G_{z x} \hat{x}+\hat{z} G_{z y} \hat{y}+\hat{z} G_{z z} \hat{z} \\
& \vec{G}=\frac{1}{(2 \pi)^{2}} \int_{-\infty}^{\infty} \int_{-\infty}^{\infty} \overrightarrow{\tilde{G}}\left(\xi, \eta, z \mid z^{\prime}\right) e^{j \xi x} e^{j \eta y} d \xi d \eta
\end{aligned}
$$

where (for $\beta=x, y$ )

$$
\begin{aligned}
& \tilde{G}_{\beta \beta}\left(\xi, \eta, z \mid z^{\prime}\right)=\frac{e^{-p_{a}\left|z-z^{\prime}\right|}}{2 p_{a}} \\
& +\frac{\varepsilon p_{a} \psi_{a}\left[\operatorname{ch} p_{a}\left(z+z^{\prime}-\delta\right)+e^{-p_{a} \delta} \operatorname{ch} p_{a}\left(z-z^{\prime}\right)\right]}{2 p_{a}\left(\varepsilon p_{a} \psi_{a}+\varepsilon_{0} p_{m} \psi_{m}\right) \operatorname{sh} p_{a} \delta} \\
& -\frac{\varepsilon_{0} p_{m} \psi_{m}\left[\operatorname{sh} p_{a}\left(z+z^{\prime}-\delta\right)+e^{-p_{a} \delta} \operatorname{ch} p_{a}\left(z-z^{\prime}\right)\right]}{2 p_{a}\left(\varepsilon p_{a} \psi_{a}+\varepsilon_{0} p_{m} \psi_{m}\right) \operatorname{ch} p_{a} \delta} \\
& +\frac{\tilde{G}_{z z}\left(\xi, \eta, z \mid z^{\prime}\right)=\frac{e^{-p_{a}\left|z-z^{\prime}\right|}}{2 p_{a}}}{2 p_{a}\left(p_{m} \psi_{a}+p_{a} \psi_{m}\right) \operatorname{sh} p_{a} \delta} \\
& -\frac{p_{a} \psi_{m}\left[e^{-p_{a} \delta} \operatorname{ch} p_{a}\left(z-z^{\prime}\right)-\operatorname{sh} p_{a}\left(z+z^{\prime}-\delta\right)\right]}{2 p_{a}\left(p_{m} \psi_{a}+p_{a} \psi_{m}\right) \operatorname{ch} p_{a} \delta}
\end{aligned}
$$

and

$$
\begin{aligned}
& \tilde{G}_{z\left\{\begin{array}{l}
x \\
y
\end{array}\right\}}\left(\xi, \eta, z \mid z^{\prime}\right)=\left\{\begin{array}{l}
j \xi \\
j \eta
\end{array}\right\} . \\
& \frac{\varepsilon \psi_{a} \psi_{m}\left(k_{0}^{2}-k^{2}\right)\left[\operatorname{sh} p_{a}\left(z-z^{\prime}\right)+\operatorname{sh} p_{a}\left(z+z^{\prime}\right)\right]}{\left(\varepsilon p_{a} \psi_{a}+\varepsilon_{0} p_{m} \psi_{m}\right)\left(p_{m} \psi_{a}+p_{a} \psi_{m}\right) k^{2} \operatorname{sh} 2 p_{a} \delta}
\end{aligned}
$$

with $\operatorname{ch} x=\cosh x, \operatorname{sh} x=\sinh x$ and

$$
\psi_{a}=\operatorname{sh} p_{a} \delta \operatorname{ch} p_{m} d, \psi_{m}=\operatorname{ch} p_{a} \delta \operatorname{sh} p_{m} d .
$$

Note that by letting $p_{a}=p_{m}, \varepsilon_{0}=\varepsilon$, and $\delta=0,(18)$ -(22) reduce to the published dyadic Green's function for a magnetically excited, single-layer, parallel-plate waveguide [8].

\section{EXPERIMENTAL RESULTS}

In this section, the effects of lift-off error in dualprobe material characterization measurements are investigated. In addition, the theoretical analysis of
Sections 2-3 is utilized to demonstrate the effectiveness of lift-off error mitigation. It is assumed here that only the $T E_{10}$ dominant mode is implemented in the modal expansion in the MoM solution. The accommodation of higher-order modes is the topic of future research.

The permittivity and permeability results of an electromagnetic shielding material (Eccosorb FGM$125)$ having thickness $d=0.3175 \mathrm{~cm}(0.125 \mathrm{in})$ and gap error $\delta=0.0635 \mathrm{~cm}(0.025 \mathrm{in})$ are shown in Figures 2 and 3 , respectively. The baseline zero airgap data is shown in black. The blue traces represent the uncorrected data extraction (two-layer Green's function not applied) for the given air gap and the red traces represent the corrected data extraction (twolayer Green's function applied) for the given air gap. The root-mean-squared error (RMSE) results clearly show the validity and benefit of the two-layer Green's function air-gap mitigation analysis. It is anticipated that the inclusion of higher-order modes will significantly improve the results. Note, other gap scenarios showed similar trends as the data presented here, but were not included for the sake of brevity.

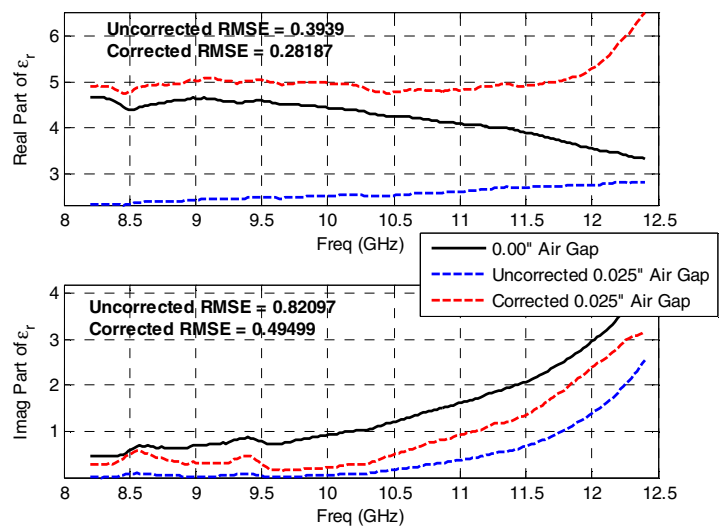

Figure 2: Permittivity of FGM-125 $(\delta=.0635 \mathrm{~cm})$.

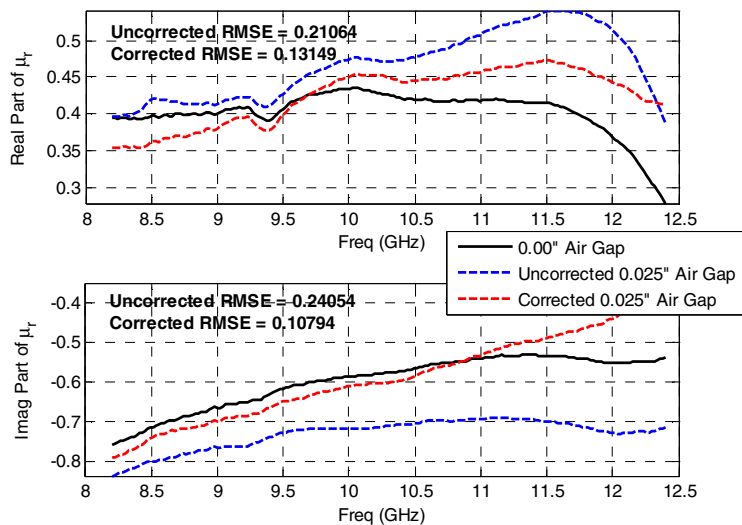

Figure 3: Permeability of FGM-125 ( $\delta=.0635 \mathrm{~cm})$. 


\section{CONCLUSION}

The effects of lift-off (i.e., air-gap) error in dual probe material characterization measurements were investigated in this paper. The primary contribution was in the mitigation of this air-gap error using a rigorous MFIE formulation based upon Love's equivalence principle and a two-layer dyadic Green's function analysis. It was experimentally demonstrated that the air-gap mitigation analysis significantly improved the material parameter extraction results when compared to the single-layer uncorrected results ( $T E_{10}$ mode only considered). It is anticipated in future research that the inclusion of higher-order modes will have an important impact in improving the air-gap mitigation results.

\section{References}

[1] C. Chang, K. Chen, and J. Qian, "Nondestructive determination of electromagnetic parameters of dielectric materials at X-band frequencies using a waveguide probe system," IEEE Trans. Instrum. Meas., vol. 46, no. 5, pp. 1084-1092, Oct. 1997.

[2] F. Mazlumi, S. Sadeghi, and R. Moini, "Interaction of rectangular open-ended waveguides with surface tilted long cracks in metals," IEEE Trans. Instrum. Meas., vol. 55, no. 6, pp. 2191-2197, Dec. 2006.

[3] D. Popovic et al, "Precision open-ended coaxial probes in in vivo and ex vivo dielectric spectroscopy of biological tissues at microwave frequencies," IEEE Trans. Microwave Theory Tech., vol. 53, no. 5, pp. 1713-1722, May 2005.

[4] J. W. Stewart and M. J. Havrilla, "Electromagnetic characterization of a magnetic material using an open-ended waveguide probe and a rigorous full-wave multimode model," Journal of Electromagnetic Waves and Applications, vol. 20, no. 14, pp. 2037-2052, 2006.

[5] J. W. Stewart and M. J. Havrilla, "A novel method for simultaneously extracting electric and magnetic properties of shielding materials using two coupled collinear open-ended waveguides," in 23rd Annual Review of Progress in Applied Computational Electromagnetics, Verona, Italy, Mar. 19-23, 2007.

[6] M. W. Hyde IV et al, "Nondestructive electromagnetic material characterization using a dual waveguide probe: a full wave solution," Radio Science, accepted, 2009.

[7] R. E. Collin, Field Theory of Guided Waves, 2nd Ed., IEEE Press, New York, 1991.

[8] G. Hanson and A. Yakovlev, Operator Theory for Electromagnetics: An Introduction, SpringerVerlag, New York, 2001. 